\title{
Concealed congenital long QT syndrome during velopharyngeal dysfunction correction: a case report
}

\author{
Soeun Jeon ${ }^{1,2}$, Hyeon-Jeong Lee ${ }^{1,2 *}$, Young-hoon Jung ${ }^{1}$, Wangseok Do ${ }^{1}$, Ah-Reum Cho ${ }^{1}$, Jiseok Baik ${ }^{1}$, \\ Do-Won Lee', Eun-Jung Kim³, Eunsoo Kim', Jeong-Min Hong ${ }^{1}$ \\ 'Department of Anesthesia and Pain Medicine, School of Medicine, Pusan National University, Busan, Korea \\ ${ }^{2}$ Medical Research Institute, Pusan National University Hospital, Busan, Korea \\ ${ }^{3}$ Department of Dental Anesthesia and Pain Medicine, School of Dentistry, Pusan National University, Dental Research Institute, Yangsan, \\ Korea
}

\begin{abstract}
The congenital long QT syndrome (LQTS) is an inherited cardiac disorder characterized by increased QT intervals and a tendency to experience ventricular tachycardia, which can cause fainting, heart failure, or sudden death. A 4-year-old female patient undergoing velopharyngeal correction surgery under general anesthesia suddenly developed Torsades de pointes. Although the patient spontaneously resolved to sinus rhythm without treatment, subsequent QT prolongation persisted. Here, we report a case of concealed LQTS with a literature review.
\end{abstract}

Keywords: Electrocardiogram; Long QT Syndrome; Torsades de Pointes.

This is an Open Access article distributed under the terms of the Creative Commons Attribution Non-Commercial License (http://creativecommons.org/licenses/by-nc/4.0/) which permits unrestricted non-commercial use, distribution, and reproduction in any medium, provided the original work is properly cited.

\section{INTRODUCTION}

QT interval prolongation on the electrocardiogram (ECG) is a rare condition in which the repolarization of the heart is delayed. Congenital long QT syndrome (LQTS) is a cardiac ion channel disease (channelopathies) and a high proportion of untreated patients end up with cardiac arrest and sudden death [1]. Although symptomatic congenital LQTS is characterized by recurrent syncope, cardiac arrest, and seizures, only $60 \%$ of patients are symptomatic at the time of diagnosis [2]. Congenital LQTS commonly presents with normal corrected QT (QTc) interval especially in the early years of life and asymptomatic carriers of the gene. Moreover, LQTS patients are known to be at risk of developing life- threatening arrhythmias after exposure to several medications such as anesthetics, antibiotics, and decongestants [3]. Clinical identification of LQTS is difficult in asymptomatic LQTS patients with normal QTc. The diagnosis of LQTS based on family history and electrophysiologic criteria such as QTc may be unreliable especially in variants of the disease and asymptomatic carriers [4]. Approximately $25-40 \%$ of patients with genetically proven congenital LQTS have a nondiagnostic resting QTc (“concealed LQTS") [5]. To date, there are still no established gold-standard diagnostic methods.

We report a case of concealed congenital LQTS newly manifested during velopharyngeal dysfunction correction with a literature review.

Received: April 8, 2020 - Revised: May 7, 2020 - Accepted: May 12, 2020

Corresponding Author: Hyeon-Jeong Lee, Department of Anesthesia and Pain Medicine, School of Medicine, Pusan National University, 179 Gudeok-ro, Seo-gu,

Busan 49241, Korea

Tel: +82-51-240-7399 Fax: 82-51-242-7466 E-Mail: Ihjksk@pusan.ac.kr

Copyright(C) 2020 Journal of Dental Anesthesia and Pain Medicine 


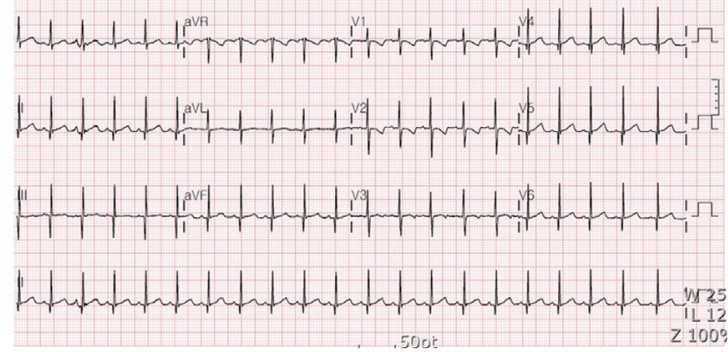

a. At birth (HR:125 bpm, QTc: $445 \mathrm{~ms}$ )

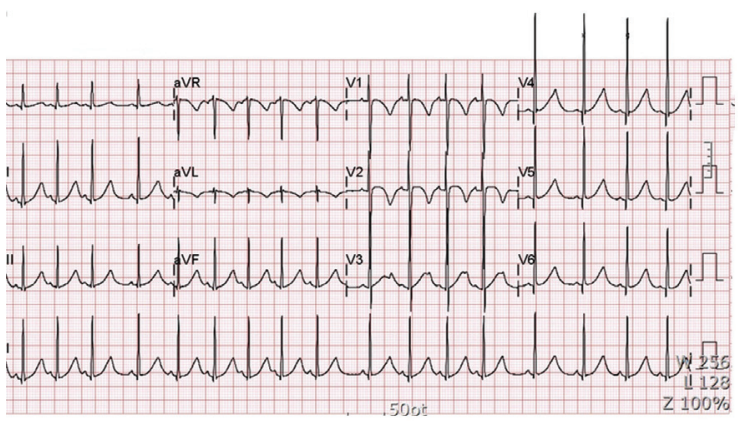

c. During operation after TdP (HR:105 bpm, QTc: $556 \mathrm{~ms}$ )

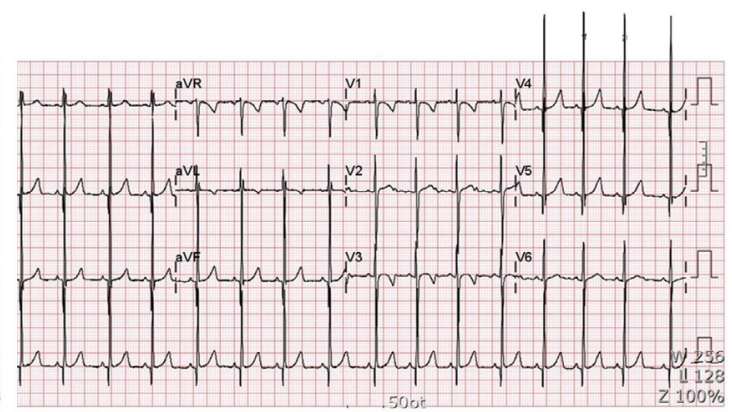

b. Pre-operation (HR:94 bpm, QTc: $445 \mathrm{~ms}$ )
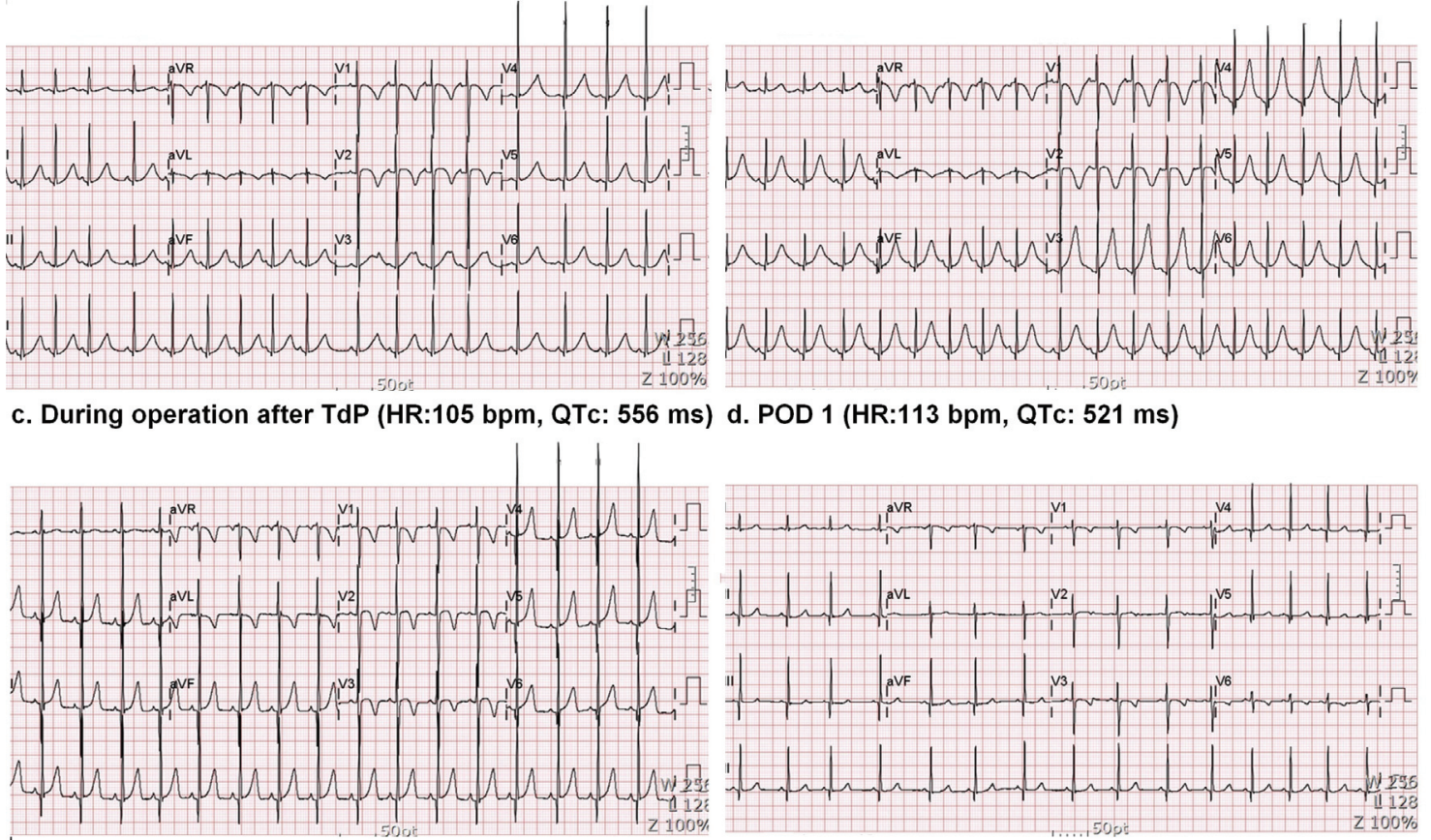

d. POD 1 (HR:113 bpm, QTc: $521 \mathrm{~ms}$ )

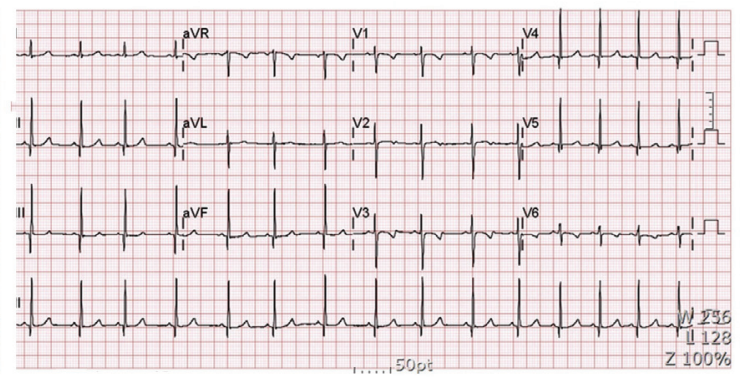

e. POD 5 after $\beta$ blocker use (HR:101 bpm, QTc: 457 ms)

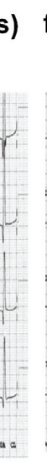

g. Before epinephrine stress test (7 Month later) (HR: 91 bpm, QTc: 439 ms)

Fig. 1. Electrocardiogram. QTc is significantly prolonged at $\mathrm{c}$ and $\mathrm{d}$.

\section{CASE REPORT}

A 4-year-old female patient weighing $22 \mathrm{~kg}$ was admitted for treatment of velopharyngeal dysfunction and programmed for general anesthesia. She had undergone cleft palate surgery at the age of 10 months, but at that time, there was no event during the administration of general anesthesia. She had no history of bradycardia, syncope, or palpitation. Physical examination was normal. 


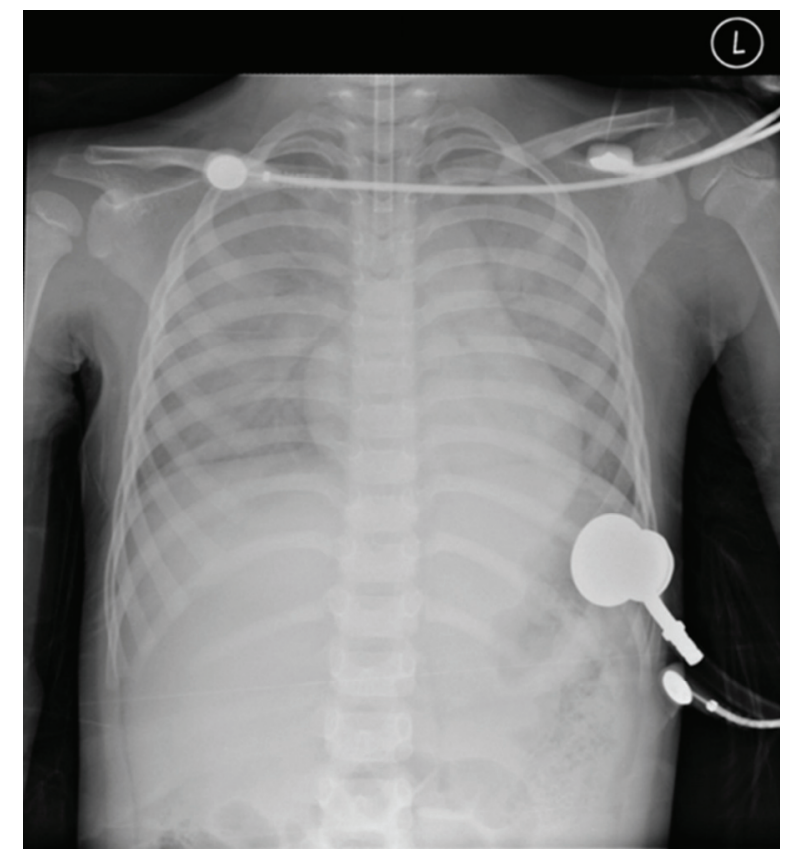

Fig. 2. Postoperative chest X-ray. Mild diffuse pulmonary edema of both lung field and mild thickening of right major fissure with right pleural effusion were seen.

Laboratory investigations revealed a sodium level of 140 $\mathrm{mmol} / \mathrm{L}$ (the normal range $135-145 \mathrm{mmol} / \mathrm{L}$ ), a potassium level of $4.6 \mathrm{mmol} / \mathrm{L}$ (the normal range $3.5-5$ $\mathrm{mmol} / \mathrm{L}$ ), $\mathrm{Cl} 102.4 \mathrm{mmol} / \mathrm{L}$, and ionized calcium level of $1.31 \mathrm{mmol} / \mathrm{L}$ with a normal albumin level. Her chest $\mathrm{X}$-ray was normal. The ECG of the patient at birth is presented in Fig. 1a. On baseline ECG, heart rate (HR) was 94 beats/min with normal sinus rhythm (Fig. 1b). Borderline QTc interval prolongation [3] of $445 \mathrm{~ms}$ (all corrected QT intervals were calculated using the Bazett correction formula, Fig. 1) was observed without $\mathrm{T}$ wave abnormality.

On arriving operating room, standard monitoring devices were used. Anesthesia was induced using thiopental $100 \mathrm{mg}$ and rocuronium bromide $20 \mathrm{mg}$ intravenously. After tracheal intubation, the anesthesia was maintained with sevoflurane. After tracheal intubation, the patient was ventilated with oxygen and air $\left(\mathrm{FiO}_{2}, 50 \%\right)$, and the end-tidal $\mathrm{CO}_{2}$ was maintained between 35 and $40 \mathrm{mmHg}$. For hemostasis and a clear local field, $1 \mathrm{ml}$ of $1 \%$ lidocaine with $1: 100,000$ epinephrine solution (approximately $10 \mu \mathrm{g}$ of epinephrine) was injected in the soft palate and posterior part of the pharynx by the surgeon. Suddenly, her blood pressure (BP) increased from $100 / 60$ to $150 / 70 \mathrm{mmHg}$, HR also increased from 113 to $175 \mathrm{bpm}$, and Torsades de pointes (TDP) was detected. Her HR was then 200-210 bpm and her BP was unmeasurable during TDP. Pulse oximetry could not detect the peripheral oxygen saturation and a cyanotic blood color was detected by the surgeon. After stopping sevoflurane, we ventilated the patient with $100 \%$ oxygen. While preparing for cardiac resuscitation and medication, TDP was converted to normal sinus rhythm without medication and chest compression/defibrillation after about 60 seconds. BP was $82 / 42 \mathrm{mmHg}$ and dopamine $4.0 \mathrm{ug} / \mathrm{kg} / \mathrm{min}$ were administered. After confirming that the patient's vital signs were stable for 10 minutes, the planned surgery proceeded under sevoflurane anesthesia. QT prolongation $(\mathrm{QTc}=556 \mathrm{~ms}$, Fig. 1c) continued until the end of the surgery. During the rest of the period, the operation went on without any events. After confirming consciousness and spontaneous breathing, the patient was admitted to the surgical intensive care unit.

Postoperative laboratory findings were within the normal range as follows: sodium level of $139.1 \mathrm{mmol} / \mathrm{L}$ (the normal range 135-145 mmol/L), a potassium level of $4.43 \mathrm{mmol} / \mathrm{L}$ (the normal range $3.5-5 \mathrm{mmol} / \mathrm{L}$ ), $\mathrm{Cl} 103.4$ $\mathrm{mmol} / \mathrm{L}$, magnesium $0.83 \mathrm{mmol} / \mathrm{L}$, and ionized calcium level of $1.25 \mathrm{mmol} / \mathrm{L}$. Transthoracic echocardiography showed normal systolic function, but chest X-ray revealed pulmonary edema (Fig. 2). The change in QTc and T wave alternans was demonstrated in Fig. 1c. The patient was referred to the pediatric department and diagnosed with a high probability of LQTS following Schwartz criteria with a score of 5 (QTc $>480 \mathrm{~ms}$, TDP, and T wave alternans). The pediatrician prescribed the propranolol $10 \mathrm{mg}$ ter in die and QT interval and chest X-ray findings were normalized 5 days after the event (Fig. 1d and 1e). The patient was discharged after planning of genetic workup and prophylactic propranolol was maintained for 3 months. The ECG of the patient 4 months after surgery is presented in Fig. 1f. In the family workup, her parents did not have any history of 
arrhythmia, but her grandfather had an undefined arrhythmia with cardiac pacemaker insertion. Genetic testing was conducted only for LQT1 (KCNQ1) and showed a negative result. No other genetic tests were performed with parental rejection due to the high cost. Seven months later, the patient underwent an epinephrine QT stress test. Even at $0.15 \mu \mathrm{g} / \mathrm{kg} / \mathrm{min}$ infusion rate of epinephrine, her response was negative (QTc $=437 \mathrm{~ms}$, Fig. $1 \mathrm{~g}$ and $1 \mathrm{~h})$. Her parents refused any further evaluation. In the subsequent months of observations, the patient reported no obvious events.

\section{DISCUSSION}

LQTS is a malfunction of cardiac ion channels resulting from mutations involving genes that code for critical ion channels of the heart (congenital LQTS), or caused by metabolic abnormalities, or drugs (acquired LQTS) [5]. Congenital LQTS may manifest anytime from infancy to old age. In this case, concealed congenital LQTS was unmasked after exposure to complex stimuli such as anesthesia, operative stress, and QT-prolonging medications in an asymptomatic 4-year-old pediatric patient.

LQTS occurs in about one out of 5,000 people and might be asymptomatic for life or cause fainting, seizures, or sudden death at a young age. LQTS usually occurs due to prolongation of action potential duration and increased susceptibility to TDP, ventricular fibrillation, and sudden cardiac death [6]. The "short-long-short" sequence heralding TDP is the hallmark of both congenital and acquired LQTS. TDP usually reverts spontaneously to sinus rhythm in most instances but degenerates to ventricular fibrillation in others.

LQTS might be diagnosed by the QTc interval on ECG, clinical symptoms, and family history. However, many patients with normal QTc genetically unproven LQTS have the possibility of being the 'concealed LQTS' patients. Thus, the diagnosis of LQTS is challenging. The Schwartz criteria are commonly used as a diagnostic tool
[7], however, one-third of the patients do not meet the diagnostic criteria at the time of diagnosis. In some patients with suspected LQTS who are not diagnosed with the Schwartz criteria and ECG, genetic testing or the epinephrine QT stress test might be needed.

Till date, genetic testing that identifies diagnostic changes in one or more of the 17 genes known to be associated with LQTS - of which KCNH2 (LQTS2), KCNQ1 (locus name LQTS1), SCN5A (LQTS3), KCNE1 (LQT5), and KCNE2 (LQT6) are most common $[8,9]$. The LQT1, LQT2, and LQT3 genotypes account for an estimated $70 \%$ to $75 \%$ of LQTS, and the majority of genotype-phenotype relationships pertain to these 3 LQTS genotypes [8]. The epinephrine QT stress test can unmask concealed type 1-LQTS (KCNQ1) with a high degree of accuracy [10,11]. Normally, enhanced sympathetic activity shortens the ventricular action potential duration and QT interval whereas increased sympathetic activity can prolong the QT interval in LQTS 1 and 2 . However, there is some variation in the degree of response to sympathetic activation depending on the type of LQTS [12].

In this case, the young female patient had the prolonged QT interval and the self-terminated TDP after injection of $10 \mu \mathrm{g}$ of epinephrine into the surgical field. In LQTS, cardiac events are uncommon after the age of 40 years; when present, they are often triggered by administration of a QT-prolonging drug, hypokalemia, or are associated with the LQTS type 3 phenotype [4]. We suspected congenital LQTS after consideration of the patient's age, family history, QT prolongation in ECG for 5 days even after the effects of epinephrine disappeared and improvement with propranolol medication. Although we clinically diagnosed the patient with congenital LQTS, no genetic mutations were found in the KCNQ1 genetic test and no further genetic tests were performed due to parental refusal. Moreover, the epinephrine QT stress test showed a negative result. Although the epinephrine QT test has been shown to be a powerful tool to predict the genotype of LQTS 1, in other types of LQTS, more than $50 \%$ of patients showed a negative response to 
epinephrine [13]. Thus, the patient can be diagnosed with congenital LQTS because she meets the criteria, but the diagnosis is not LQTS 1 because the KNCQ1 gene and epinephrine stress test were negative.

Anesthesiologists should consider the possibility of the presence of LQTS and special attention to ECG changes during anesthesia is needed. These subsets of patients are highly prone to various ventricular arrhythmias and sudden cardiac death and hence should be evaluated and treated to normalize the QT interval, prior to anesthetic exposure.

Moreover, phenotypically mild (subclinical) mutations or polymorphisms in LQTS genes could be present in the general population, and it has been suggested that these patients could be predisposed to drug-induced or hypokalemia-induced arrhythmias.

When QT-prolongation-inducing drugs were administered or during other conditions favoring QT prolongation such as bradycardia or hypokalemia, the "repolarization reserve" was exhausted, leading to QT prolongation and TDP. Therefore, the molecular genetic analysis might be extended to those with acquired LQTS and their family.

Some anesthetic agents like sevoflurane, thiopentone, and other drugs are known to prolong the QT interval, the strength of association in precipitating TDP and ventricular fibrillation in these patients is unknown.

Flurane can affect cardiac repolarization and cause LQTS, which is common in patients with old age, electrolyte abnormalities, and ischemic heart disease [14]. Volatile anesthetics such as halothane, enflurane, isoflurane, and sevoflurane could prolong the QT interval to some extent, however, they have been successfully used in patients with congenital LQTS receiving beta-blocker therapy $[15,16]$.

Thiopental prolongs the QT interval in healthy patients. Despite this effect, thiopental has been used safely in patients with congenital LQTS. Because of its prolongation of the action potential duration, thiopental may in fact reduce the heterogeneity of action potential dispersion through the ventricular wall (i.e., reduce transmural dispersion of repolarization). This effect would theoretically prevent the spontaneous onset of TDP $[17,18]$. Glycopyrrolate has also been shown to lengthen the QT interval in healthy subjects [19].

A case of sevoflurane-associated TDP in a LQTS 2 patient was reported [20]. Pulseless ventricular tachycardia was sustained and ended after defibrillation in the patient. In LQTS 2, the risk of sudden death is reportedly high. As in other cases, TDP in our case was triggered after sevoflurane use and epinephrine injection, however, it ended automatically a minute after stopping sevoflurane and $100 \%$ oxygen ventilation, featured as a mild form.

Current evidence suggests that $5-10 \%$ of persons in whom TDP develops on exposure to QT-intervalprolonging drugs conceal mutations associated with LQTS and can, therefore, be viewed as having a subclinical form of the congenital syndrome.

$\beta$-Blockade is the first-line treatment LQTS with a resting QTc greater than $470 \mathrm{~ms}$. Moreover, in asymptomatic patients with LQTS having resting QTc less than $470 \mathrm{~ms}$, prophylactic treatment with $\beta$-Blockade is recommended to reduce the risk of event recurrence [21,22].

In the case of TDP, acute therapy should follow advanced cardiovascular life support guidelines. According to the 2015 American Heart Association (AHA) Pediatric Advanced Life Support (PALS) guidelines, wide Complex Tachycardia (QRS > 0.09 secs) with hypotension, altered level of consciousness, or signs of shock requires immediate synchronized cardioversion [23]. Because of the polymorphic nature of TDP, synchronized cardioversion may not be possible, and the patient may require unsynchronized defibrillation [22].

Magnesium sulfate $(2 \mathrm{~g})$ should also be administered intravenously to shorten the QT interval and potentially stabilize the cardiac cell membranes from early after depolarization-type premature ventricular complexes, which can promote TDP [23]. As a nonpharmacological treatment, implantable cardioverter-defibrillator (ICD) therapy could be considered in patients with congenital LQTS and history of aborted cardiac arrest, recurrent 
cardiogenic syncopal events despite $\beta$-blockade or patients who cannot tolerate medical therapy [24-26].

In conclusion, anesthesiologists should be aware of the presence of QT prolongation in preoperative ECG and prepare for TDP and cardiac arrest if QT prolongation observed in the perioperative period. Several drugs that prolong the QT interval should be cautiously used. Genetic workup or epinephrine QT stress test for an accurate diagnosis of LQTS might be needed after surgery.

\section{AUHOR ORGIDS}

Soeun Jeon: https://orcid.org/0000-0002-4009-6321

Hyeon-Jeong Lee: https://orcid.org/0000-0002-3450-865X

Young-hoon Jung: https://orcid.org/0000-0001-6802-2028

Wangseok Do: https://orcid.org/0000-0003-2338-9648

Ah-Reum Cho: https://orcid.org/0000-0002-7108-1384

Jiseok Baik: https://orcid.org/0000-0003-4904-7400

Do-won Lee: https://orcid.org/0000-0001-5140-2358

Eun-Jung Kim: https://orcid.org/0000-0003-4982-9517

Eunsoo Kim: https://orcid.org/0000-0001-9978-4973

Jeong-Min Hong: https://orcid.org/0000-0003-3527-555X

\section{AUHOR GONIRIBUIONS}

Soeun Jeon: Writing - original draft

Hyeon-Jeong Lee: Conceptualization, Writing - original draft, Writing

- review \& editing

Young-hoon Jung: Investigation

Wangseok Do: Writing - review \& editing

Ah-Reum Cho: Writing - review \& editing

Jiseok Baik: Writing - review \& editing

Do-won Lee: Visualization

Eun-Jung Kim: Writing - review \& editing

Eunsoo Kim: Writing - review \& editing

Jeong-Min Hong: Resources

FOOTNOTE: This case report followed the CARE guidelines for case reports. The Hospital Institutional Review Board (IRB) designated this project as exempt (IRB number: H-2004-002-089). Written informed consent was obtained from the patient's guardian for publication of this case report. At the time of consent, the patient had recovered from her illness.

DECLARATION OF INTERESTS: All authors declare that they have no competing interests.

FUNDING: This work was supported by a 2-Year Research Grant from the Pusan National University

\section{REFERENCES}

1. Bohnen MS, Peng G, Robey SH, Terrenoire C, Iyer V, Sampson KJ, et al. Molecular pathophysiology of congenital long QT syndrome. Physiol Rev 2017; 97: 89-134.

2. Booker PD, Whyte SD, Ladusans EJ. Long QT syndrome and anaesthesia. Br J Anaesth 2003; 90: 349-66.

3. Fazio G, Vernuccio F, Grutta G, Re GL. Drugs to be avoided in patients with long QT syndrome: focus on the anaesthesiological management. World J Cardiol 2013; 5: 87-93.

4. Alders M, Bikker H, Christiaans I. Long QT Syndrome [serial on the Internet]. 2018 Feb [2018 Feb 8]. Available from http://www.ncbi.nlm.nih.gov/books/NBK1129/

5. Kies SJ, Pabelick CM, Hurley HA, White RD, Ackerman MJ. Anesthesia for patients with congenital long QT syndrome. Anesthesiology 2005; 102: 204-10.

6. Crotti L, Celano G, Dagradi F, Schwartz PJ. Congenital long QT syndrome. Orphanet J Rare Dis 2008; 3:18.

7. Schwartz PJ, Moss AJ, Vincent GM, Crampton RS. Diagnostic criteria for the long QT syndrome. an update. Circulation 1993; 88: 782-4.

8. O'Hare M, Maldonado Y, Munro J, Ackerman MJ, Ramakrishna H, Sorajja D. Perioperative management of patients with congenital or acquired disorders of the QT interval. Br J Anaesth 2018; 120: 629-44.

9. Splawski I, Shen J, Timothy KW, Lehmann MH, Priori $\mathrm{S}$, Robinson JL, et al. Spectrum of mutations in long-QT syndrome genes. KVLQT1, HERG, SCN5A, KCNE1, and KCNE2. Circulation 2000; 102: 1178-85.

10. Vyas H, Ackerman MJ. Epinephrine QT stress testing in congenital long QT syndrome. J Electrocardiol 2006; 39(4 Suppl): S107-13. 
11. Shimizu W, Noda T, Takaki H, Nagaya N, Satomi K, Kurita T, et al. Diagnostic value of epinephrine test for genotyping LQT1, LQT2, and LQT3 forms of congenital long QT syndrome. Heart Rhythm 2004; 1: 276-83.

12. Marx SO, Kurokawa J, Reiken S, Motoike H, D'Armiento J, Marks AR, et al. Requirement of a macromolecular signaling complex for beta adrenergic receptor modulation of the KCNQ1-KCNE1 potassium channel. Science 2002; 295: 496-9.

13. Clur SA, Chockalingam P, Filippini LH, Widyanti AP, van Cruijsen M, Blom NA, et al. The role of the epinephrine test in the diagnosis and management of children suspected of having congenital long QT syndrome. Pediatr Cardiol 2010; 31: 462-8.

14. International Council of Harmonization (ICH) Harmonised Tripartite Guideline. The clinical evaluation of QT/ QTc interval prolongation and proarrhythmic potential for non-antiarrhythmic drugs E14 [serial on the Internet]. 2005 May. Available from

https:// fossaconsulting.com/wp-content/uploads/2016/ 08/ae361d1b023c176a97a14030f4063b39.pdf.

15. Abe K, Takada K, Yoshiya I. Intraoperative torsade de pointes ventricular tachycardia and ventricular fibrillation during sevoflurane anesthesia. Anesth Analg 1998; 86: 701-2.

16. Lustik SJ, Eichelberger JP, Chibber AK, Bronsther O. Torsade de pointes during orthoptic liver transplantation. Anesth Analg 1998; 87: 300-3.

17. Medak R, Benumof JL. Perioperative management of the prolonged Q-T interval syndrome. Br J Anaesth 1983; 55: 361-4.

18. Brown M, Liberthson RR, Ali HH, Lowenstein E. Perioperative anesthetic management of a patient with long Q-T syndrome (LQTS). Anesthesiology 1981; 55: 586-9.

19. Saarnivaara L, Lindgren L. Prolongation of QT interval during induction of anaesthesia. Acta Anaesthesiol Scand 1983; 27:126-30.

20. Kumakura M, Hara K, Sata T. Sevoflurane-associated torsade de pointes in a patient with congenital long QT syndrome genotype 2. J Clin Anesth 2016; 33: 81-5.

21. Priori SG, Napolitano C, Schwartz PJ, Grillo M, Bloise R, Ronchetti E, et al. Association of long QT syndrome loci and cardiac events among patients treated with beta-blockers. JAMA 2004; 292: 1341-4.

22. Al-Khatib SM, Stevenson WG, Ackerman MJ, Bryant WJ, Callans DJ, Curtis AB, et al. 2017 AHA/ACC/HRS guideline for management of patients with ventricular arrhythmias and the prevention of sudden cardiac death: executive summary: a report of the americam college of cardiology/american heart association task force on clinical practice guidelines ande the heart rhythm society. Heart Rhythm 2018; 15: e190-252.

23. Atkins DL, Berger S, Duff JP, Gonzales JC, Hunt EA, Joyner BL, et al. Part 11: pediatric basic life support and cardiopulmonary resuscitation quality: 2015 american heart association guidelines update for cardiopulmonary resuscitation and emergency cardiovascular care. Circulation 2015; 132(18 Suppl 2): S519-25.

24. Schwartz PJ, Spazzolini C, Crotti L. All LQT3 patients need an ICD: true or false? Heart Rhythm 2009; 6: 113-20.

25. Vincent GM, Schwartz PJ, Denjoy I, Swan H, Bithell C, Spazzolini C, et al. High efficacy of beta-blockers in long-QT syndrome type 1: contribution of noncompliance and QT-prolonging drugs to the occurrence of beta-blocker treatment "failures". Circulation 2009; 119: 215-21.

26. Jons C, Moss AJ, Goldenberg I, Liu J, McNitt S, Zareba W, et al. Risk of fatal arrhythmic events in long QT syndrome patients after syncope. J Am Coll Cardiol 2010; 55: $783-8$. 